\title{
Leak detection and localization using acoustic emission technique
}

\author{
Tonphong Kaewkongka*
}

Department of Physics, Faculty of Science, Chulalongkorn University Patumwan, Bangkok 10330, Thailand

\begin{tabular}{|c|c|}
\hline ARTICLE I N F O & A B S T R A C T \\
\hline $\begin{array}{l}\text { Article history: } \\
\text { Received } 6 \text { April, } 2015 \\
\text { Accepted } 10 \text { October } 2015 \\
\text { Available online } \\
11 \text { October } 2015 \\
\text { Keywords: } \\
\text { Acoustic emission } \\
\text { Leak } \\
\text { Detection } \\
\text { Localization }\end{array}$ & $\begin{array}{l}\text { This article provides a method of acoustic emission (AE) technique to detect leakage in } \\
\text { pipeline and locate the position of the leakage. The AE sensor is made of piezoelectric effect } \\
\text { transducer to pick up the acoustic emission signal which is generated from the turbulent flow } \\
\text { at the leak position. The signal conditioning unit is used to enhanced and eliminate the } \\
\text { background noise from the leak location sources. The main acoustic emission processing unit } \\
\text { is used to acquire and process the extracted AE characteristic parameters from preprocessing } \\
\text { waveform. The leak pipeline is simulated by drilling the hole and plugged with M8 screw at } \\
\text { different locations. The results show that the proposed AE method can detect and locate } \\
\text { simultaneous leak condition in pipeline with promising results. }\end{array}$ \\
\hline
\end{tabular}

\section{Introduction}

Pipelines are the necessary part in almost all kind of petrochemical industries. Often, the pipeline rupture may be caused by the fatigue or the sudden change in pressure. This causes not only the machine failure, product loss, but also the environmental hazardous if the defected pipeline is not quickly detected and repaired. Monitoring the condition of the pipelines has obtained considerable attention over the past many years due to its particular importance for the safety and the environmental and economic reasons. A reliable condition monitoring system will significantly reduce the failure and the unplanned maintenance. Hence, the huge attendant cost due to machine down time can be significantly reduced. Over the past decades there are many kinds of methods available for the pipeline fault detection such as radio graphic testing, gradient of temperature, eddy current, flow or pressure change (Miller et al., 1999; David \& Alan 2001). However, these methods can only reveal the defect of pipeline after it was already occurred.

Acoustic emission (AE) has gained increasing credibility in recent years (Drouillard, 1996). This is largely due to the advances in acquisition speed and processor speeds required to handle the vast

\footnotetext{
* Corresponding author.

E-mail addresses: tonphong.k@chula.ac.th （T. Kaewkongka) 
amount of signal rates and events at relatively high frequencies (20 kHz-1 MHz). Acoustic emission is a natural phenomenon of stress wave generation and propagation spontaneously when a material is subjected under stress (Scruby, 1987; Pollock, 1973; Scholey et al., 2009). Plastic deformation and growth cracks are the primary sources of acoustic emission in metals. The acoustic emission signal can be detected by a piezoelectric transducer, which converts the mechanical strain energy carried by the elastic wave into an electrical signal.

The radial strain in the pipeline can be produced by the applied internal pressure, which acts upward through the thickness of the material and causes the strain. The change in strain produces the physical change around a defect which can take several forms such as the microscopic crack growth or extension, turbulent flow, the rubbing or surface crack or the corrosion crack. All defect mechanisms will generate the transient elastic stresses or acoustic emission waves that propagate along the pipeline (Kovacevich et al., 1996; Tidswell et al., 1996; Holroyd, 2002). In this study, the proposed method is to demonstrate that a condition-based monitoring using acoustic emission (AE) can provide not only timely detection of defective pipeline but also the location of defect in the pipeline so that maintenance or replacement can be performed prior to the loss of safety function. Therefore the use of acoustic emission method has been proposed to use for pipeline monitoring instead of those conventional method.

\section{The proposed approach}

Fig. 1 shows a block diagram of the proposed pipeline condition monitoring procedure. The pipeline is made from steel. The acoustic emission instrument, TITAN-914 ${ }^{T M}$, is used to acquire the acoustic emission waveform generated by turbulent flow as the leak occurred by simulated drilled hole. The length of the testing pipeline is 6 meter. The pump is used to feed the water throughout the pipeline at the pressure rate of 1.0-1.7 bar.

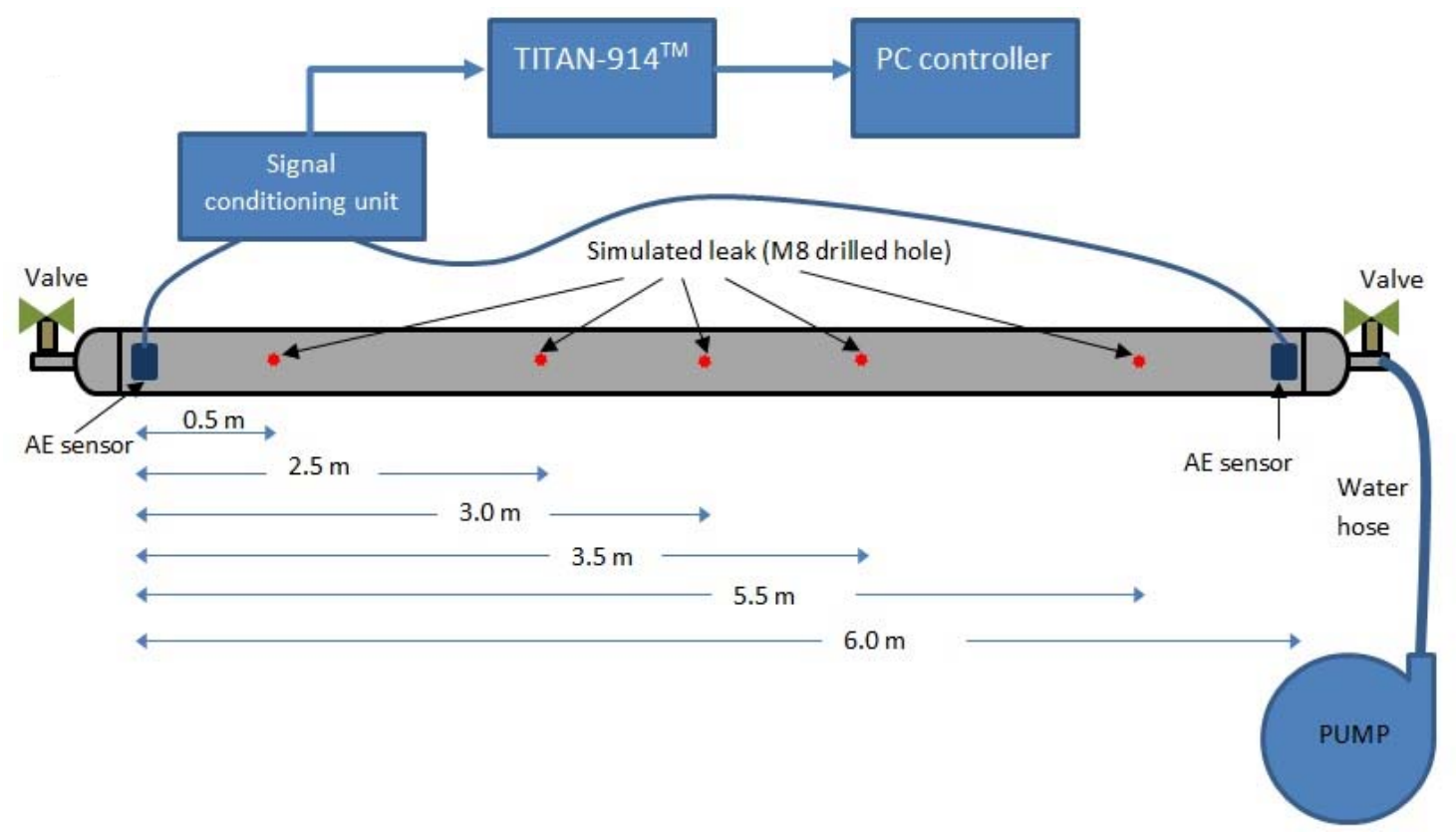

Fig. 1. The proposed pipeline leak detection block diagram

Fig. 2 shows the system configuration of TITAN-914 ${ }^{T M}$. The AE-SS1 sensors are attached to the test material. A typical 4 up to multi-channel system has DSP processing unit at high resolution so it is usable for leak testing, dynamic structural analysis, intelligent acquisition, high speed and accuracy. For remote monitoring application, a network system can be configured to generate the array of AE sensors to provide data recording in order to capture transient event and extracting of AE characteristic 
features. Those AE parameters can be correlated with the physical properties of the test material. In this study, the pipeline operating condition was monitored in various conditions: normal, single leak position, simultaneous leak positions.

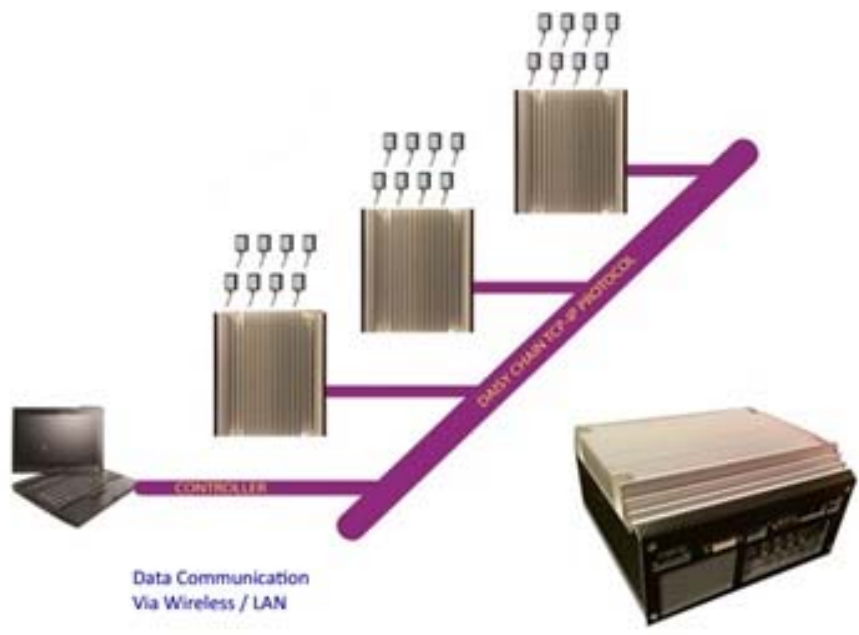

Fig. 2. TITAN-914 ${ }^{T M}$ system configuration

The AE time domain magnitude is suitable measuring its signal intensity for both burst type and continuous AE signals. Effect of signal averaging is to improve statistical significance but distorts shape of activity giving time decays and reduced peak value. It is also represented in logarithmic scale of AE signals since it can allow a greater signal range to be simultaneously observed with its unit in $\mathrm{dB}$ and is a ratio with respect to a reference voltage (Holroyd, 2002):

AE Log Magnitude in $d B=20 \log _{10}\left(V_{\text {sig }} / V_{\text {ref }}\right)$

\section{Experimental application of the proposed method}

Fig. 3 shows the configuration of the pipeline for leak detection and localization using acoustic emssion method. The AE-SS1 sensors were attached at both ends of the circumference of the pipeline. Two sensors are mounted on the circumference by means of adhesive tape. The acoustic emission wave generated from the source can be captured at resonance frequency around $100 \mathrm{kHz}$. The captured signals were then enhanced and filtered using signal condition unit. The AE processor was used to perform AE parameters extraction such as rise time, peak amplitude, counts, AE energy, inter-arrival time and etc. Those parameters are of importance in order to evaluate the condition of the pipeline. More over, the leak location can be determined using the difference of inter-arrival time between two sensors. The embeded high speed data processor is capable of processing the location of the AE generated sources.

The testing conditions are as follow:

1. No leak

2. Leaking at $3.0 \mathrm{~m}$

3. Leaking at $3.5 \mathrm{~m}$ and $5.5 \mathrm{~m}$

Acoustic emission signal generated within the pipeline was measured using AE-SS1 sensor which is manufactured by Holroyd Instruments, UK (both ends of the pipeline). The AE sensor has resonance frequency at $100 \mathrm{kHz}$. The acquired AE signals, having been band-pass filtered at $20 \mathrm{kHz}$ to $500 \mathrm{MHz}$ for noise-removal and amplified to $60 \mathrm{~dB}$, were sampled into the TITAN-914 ${ }^{\mathrm{TM}}$ system. Measurements 
were obtained from different machine conditions: no leak, leaking at $3.0 \mathrm{~m}$ and leaking at $3.5 \mathrm{~m}$ and $5.5 \mathrm{~m}$. For each condition, five signals were collected as for data processing.

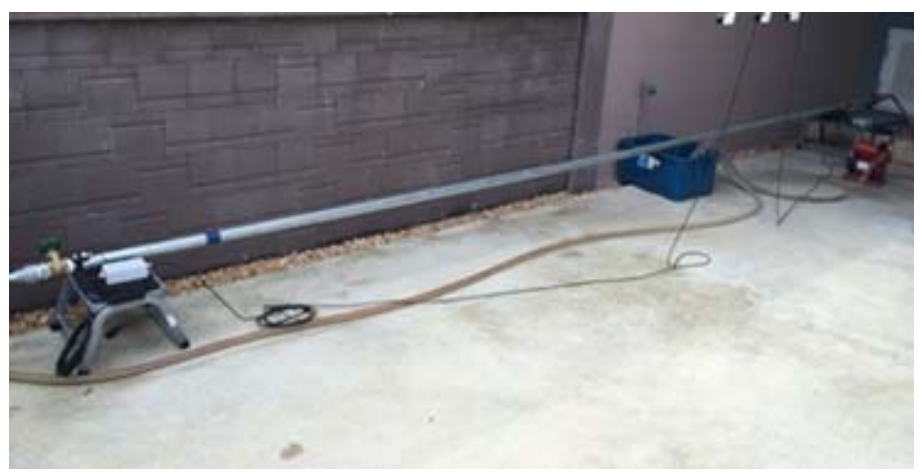

Fig. 3. Pipeline leak detection and localization experimental set up

\section{Experimental results and discussion}

\subsection{No leak condition}

The pipeline was tested as normal operating condition. Those drilled holes were plugged properly using M8 screw to ensure that there is no leak condition occurred. The time domain signals are shown in Fig. 4. The pump was operated to feed the water into the pipeline at the pressure around 1.0-1.7 bar. The AE signals were captured during the test using TITAN-914 ${ }^{T M}$. Fig. 5 illustrates the raw time domain waveforms obtained from two AE sensors. These waveforms were acquired by the transient module of TITAN-914 ${ }^{T M}$ to capture the AE activities generated during the test. At the bottom figure also shows the interact-window of the processed AE parameters from the AE channels that were activated.
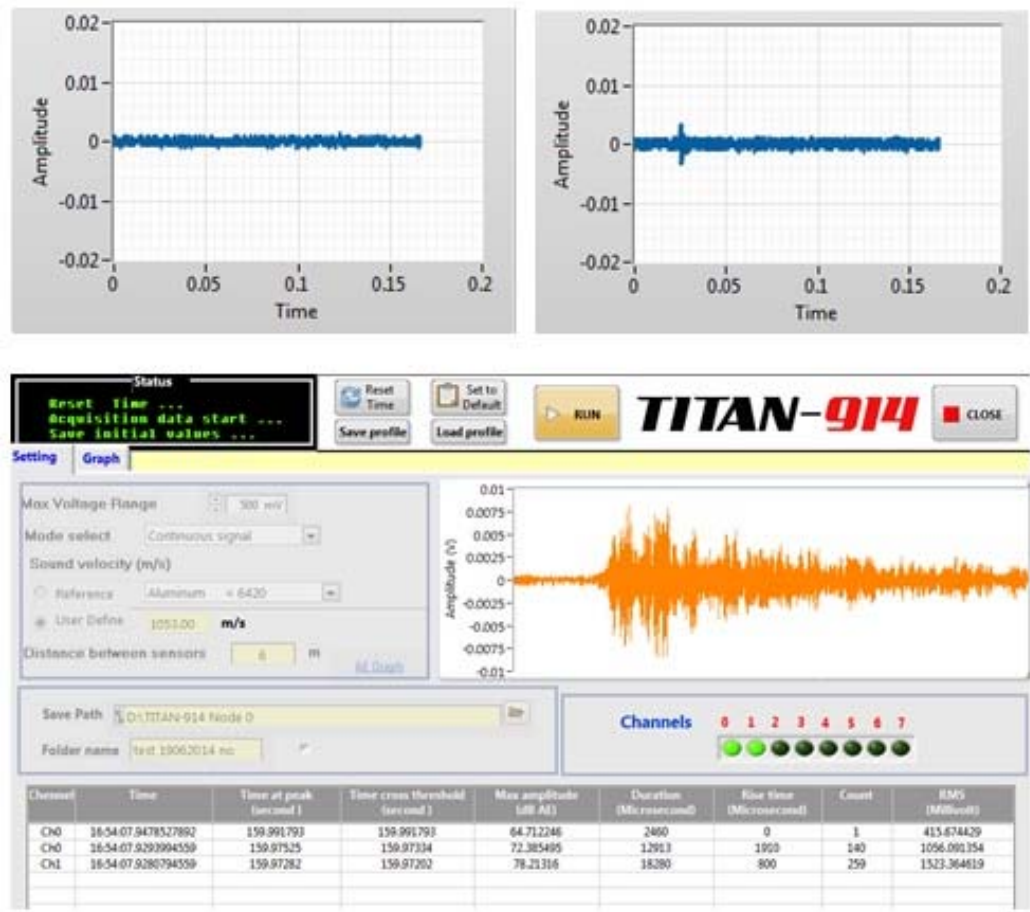

Fig. 4. Time domain waveform from no leak condition 


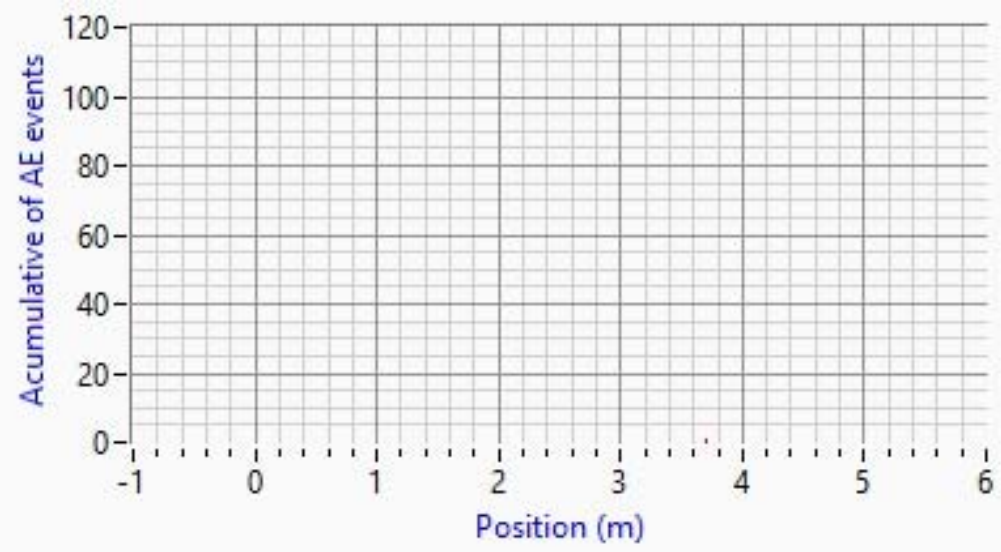

Fig. 5. Accummulative AE events versus position from no leak condition

It is obvious that with no significant $\mathrm{AE}$ activity generated during the recording period, there is no accumulated AE events versus position (in this case, the length of the pipeline as displayed in meter). Hence there is assumed that the pipeline is in normal operating condition.

\subsection{Leaking at $3.0 \mathrm{~m}$}

At the testing condition, the simulated drilled hole at location $3.0 \mathrm{~m}$ from the origin (left-most of the pipeline as shown in Fig. 1), was unplugged in order to simulate the leaking condition. The time domain signals are shown in Fig. 6. This is also recorded using Transient module of TITAN-914 ${ }^{T M}$.

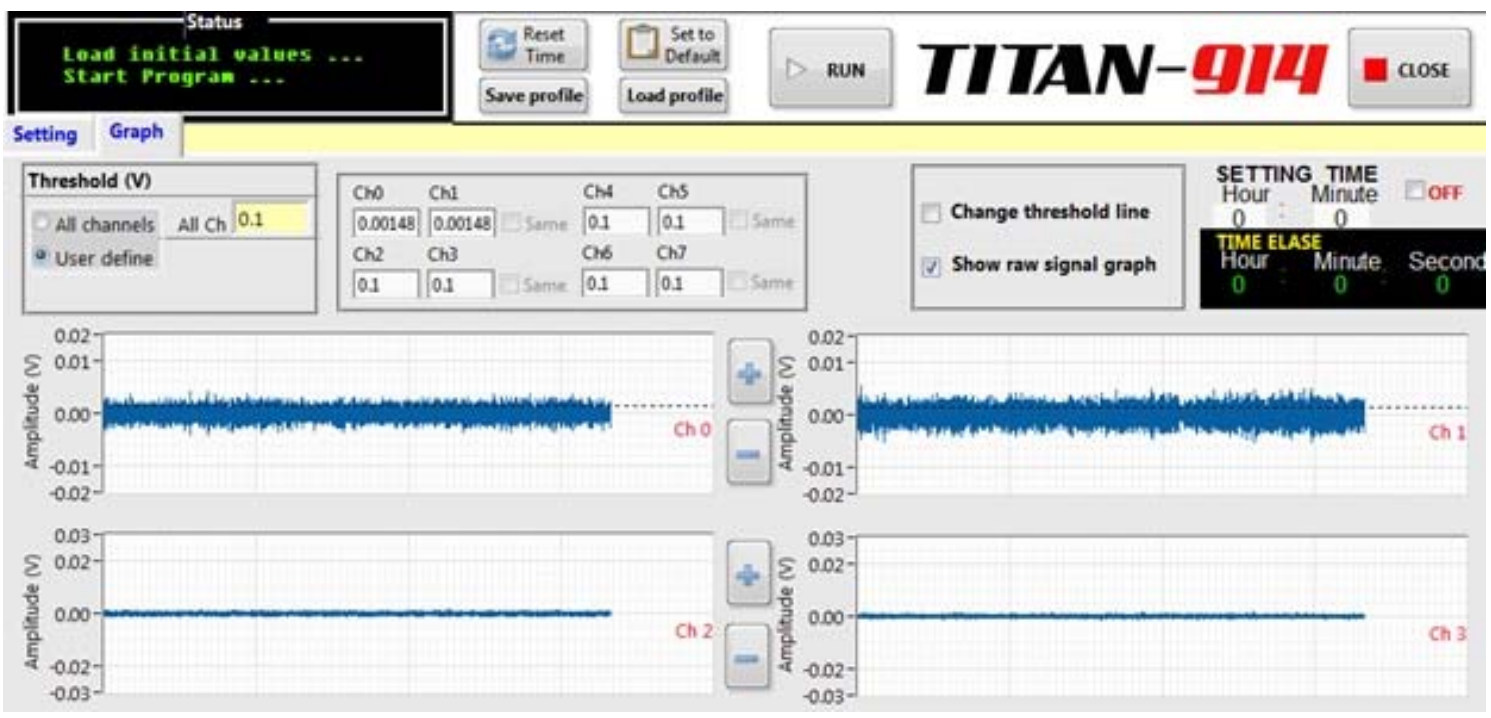

Fig. 6. Time domain waveform of the leaking location at $3.0 \mathrm{~m}$

Fig. 7 shows a plot of accumulative AE events versus position of the AE sources that occurred in the pipeline. It is clear that the position of the detected AE acitivities were accummulated at highest frequency bin around $3 \mathrm{~m}$ from the origin. The results show that the accuracy of the detection and localization of the leaking position is correct. 


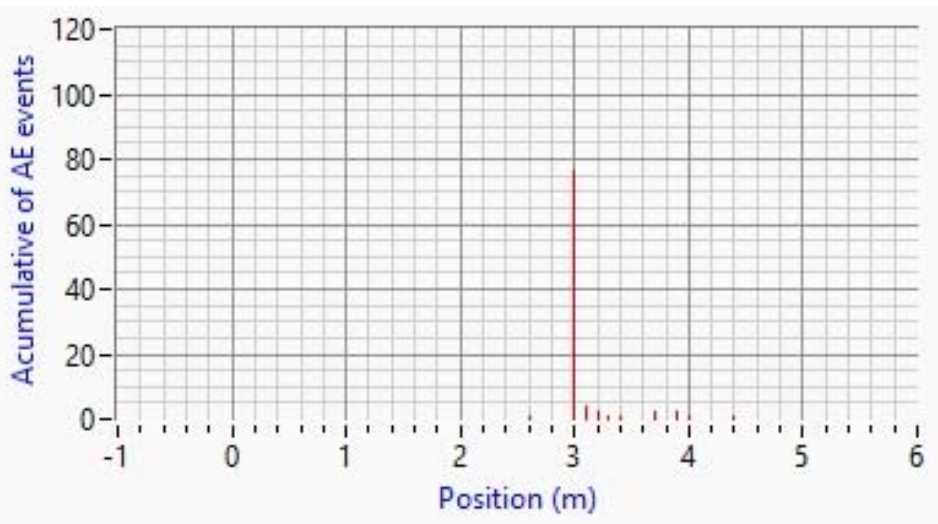

Fig. 7. Accumulative of AE events versus position from leaking at $3.0 \mathrm{~m}$

\subsection{Leaking at $3.5 \mathrm{~m}$ and $5.5 \mathrm{~m}$}

At the testing condition, the simulated drilled hole at location $3.0 \mathrm{~m}$ and $5.5 \mathrm{~m}$ from the origin (left-most of the pipeline as shown in Figure 1), were unplugged in order to simulate two simultaneous leaking conditions. The time domain signals are shown in Fig. 8. This is also recorded using Transient module of TITAN-914 ${ }^{T M}$.

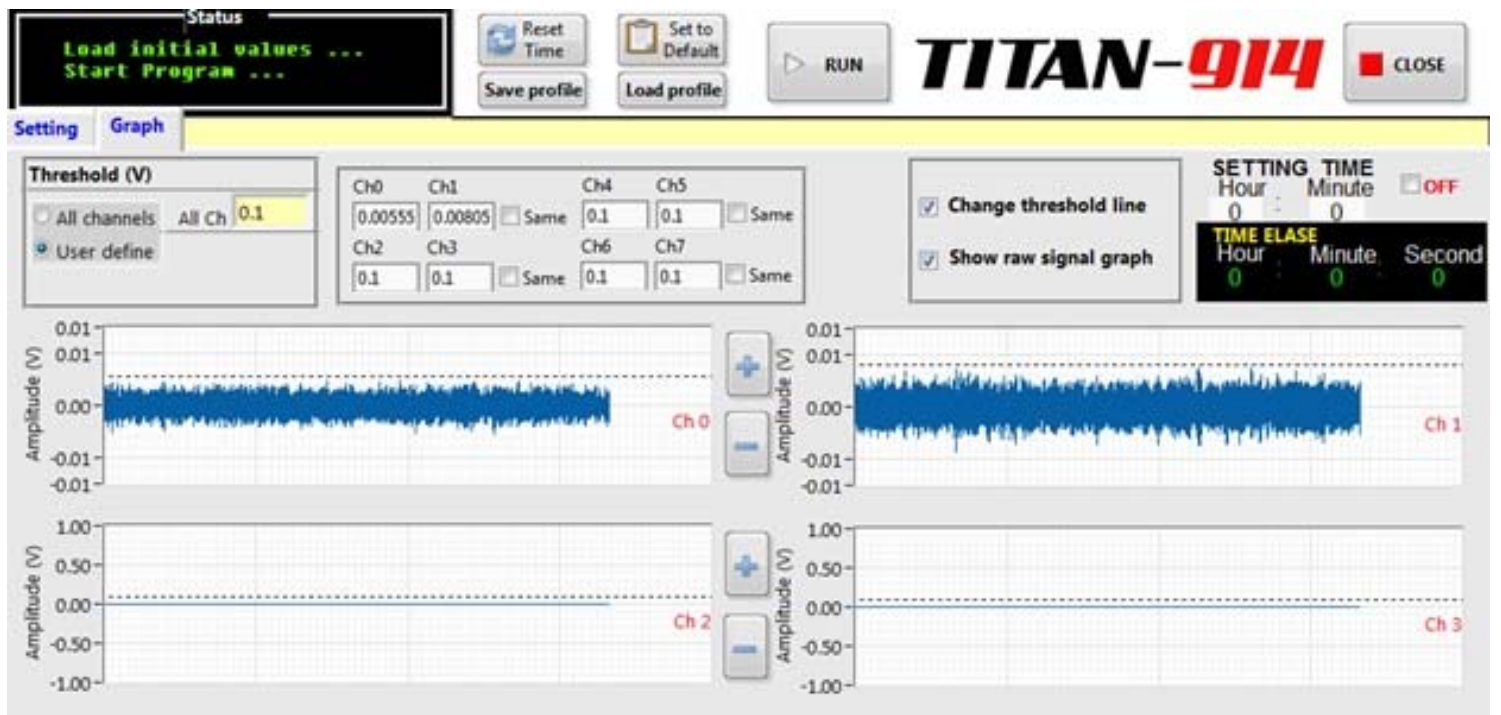

Fig. 8. Time domain waveform of the leaking location at $3.5 \mathrm{~m}$ and $5.5 \mathrm{~m}$

Fig. 9 shows a plot of accumulative AE events versus position of the AE sources that occurred in the pipeline. There were two distinct highest peak of the accumulated AE events occurred at $3.5 \mathrm{~m}$ and $4.9 \mathrm{~m}$. It is worth to note that with such simultaneous leaking condition the system can identify the AE generated sources as two distinct peaks of the AE acculation events. It is clear that the position of the detected AE acitivities were accummulated at highest frequency bin around $3.5 \mathrm{~m}$ and $4.9 \mathrm{~m}$ from the origin. Fig. 10 shows the example of the actual simulated leak of the pipleine. At the $3.5 \mathrm{~m}$ is the exact leak location as simulated drilled hole but at the actual $5.5 \mathrm{~m}$ there is some variation of the calculated position. This is due to the turbulent flow of those two simultaneous leaks can mask the signal from the other. Hence leading to the variation of the predicted position around $8 \%$. 


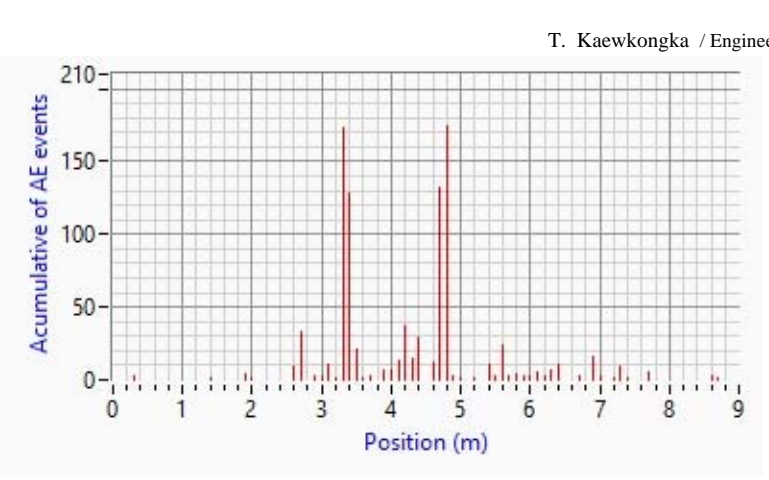

Fig. 9. Accumulative of $\mathrm{AE}$ events versus positoin from leaking at $3.5 \mathrm{~m}$ and $5.5 \mathrm{~m}$

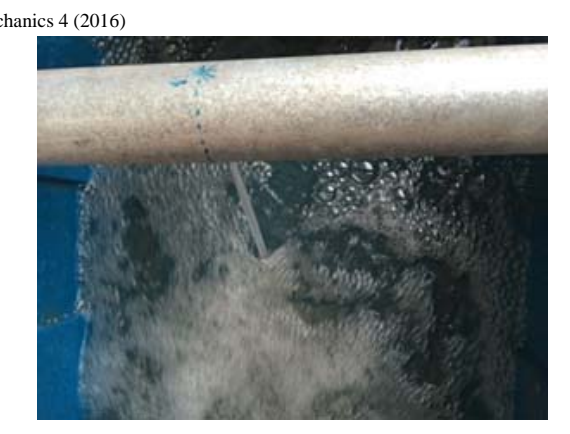

Fig. 10. Example of simulated leaking condition of the pipeline

As a results of the pipeline leak detection testing using TITAN-914 ${ }^{T M}$, the results of the system to detect the presence of leak is very promising. The system utilizes the use of AE transducers to pick up elastic wave generated inside the pipeline and propagate through the outer surface of the testing pipeline. The captured signals were then amplified and filtered using signal conditioning unit to provide the appropriate signal-to-noise ratio for further AE signal processing. Then the TITAN-914 ${ }^{T M}$ was used to provide the AE parameters which can be used to identify the presence of leak of the pipeline. The transient waveforms which are in time-domain waveform can also be captured for further in-depth diagnostics.

It was found that at the leaking point of the pipeline are of those AE activiated sources caused by the turbulent flow of the leaking water at the simulated drilled hole. The evaluation of the position of the leaking source can be obtained by the plot of accummulative AE events generated versus position of the pipeline. The leak localization of single leak location is highly accurate (as shown at $3.0 \mathrm{~m}$ ). The simultaneous leak location identification (at $3.5 \mathrm{~m}$ and $5.5 \mathrm{~m}$ ) can be revealed using two distinct peaks obtained from the plot of accumulated $\mathrm{AE}$ events versus location. The recognition rate of localization of two simultaneous leaks is of $92 \%$ accuracy.

\section{Acknowledgements}

The author gratefully acknowledges the support of the Department of Physics, Faculty of Science, Chulalongkorn University and Siam Gen Co., Ltd. for their advice and technical support.

\section{References}

David, B. C. \& Alan, E.S. (2001). State of the art for pipe and leak detection. Geophysical Survey Systems, Inc.

Drouillard, T. F. (1996). A history of acoustic emission. Journal of Acoustic Emission, 14(1), 1-34.

Holroyd, T. J. (2002). Acoustic emission sensors for OEM applications. Engineering Science and Education Journal, 11(1), 29-35.

Kovacevich, J. J., Robertson, M. O., Sanders, D. P., \& Nuspl, S. P. (1996). Recent advances in the application of acoustic leak detection to process recovery boilers. Tappi Journal, 79, 149-156.

Miller, R. K., Pollock, A. A., Watts, D. J., Carlyle, J. M., Tafuri, A. N., \& Yezzi, J. J. (1999). A reference standard for the development of acoustic emission pipeline leak detection techniques. NDT \& E International, 32(1), 1-8.

Pollock, A. A. (1973). Acoustic emission-2: acoustic emission amplitudes. Non-destructive Testing, 6(5), 264-269.

Scholey, J. J., Wilcox, P. D., Wisnom, M. R., Friswell, M. I., Pavier, M., \& Aliha, M. R. (2009). A generic technique for acoustic emission source location. Journal of Acoustic Emission, 27, 291-298.

Scruby, C. B. (1987). An introduction to acoustic emission. Journal of Physics E: Scientific Instruments, 20(8), 946-953. 
Tidswell, R. D., Shipley, M. P., \& Cane, B. J. (1996). Development of new procedures for in-service acoustic emission testing in pressure vessels and pipework. Progress in Acoustic Emission, 8, 367372. 